\title{
APOPROTEIN(a) ISOFORMS RISK FACTORS FOR ATHEROGENESIS IN ESRD PATIENTS ON HAEMODIALYSIS
}

\author{
Danica Labudovic, Katerina Tosheska-Trajkovska and Sonja Alabakovska
}

Institute of Medical and Experimental Biochemistry, University "Ss. Cyril and Methodius", Faculty of Medicine, Skopje, Republic of Macedonia

\begin{abstract}
Introduction. End-stage renal disease (ESRD) patients undergoing hemodialysis are at an increased risk of arteriosclerotic vascular disease (ASVD). The increased risk is commonly attributed to the traditional risk factors related to ESRD. However, interest for more recent risk factors for ASVD, such as the level of lipoprotein(a) and its specific apoprotein(a) has been promoted.

The aim of this paper is to determine whether apo(a) phenotype is a risk factor for arteriosclerotic vascular disease in ESRD patients who are on hemodialysis.

Methods. Apo(a) phenotypisation was performed by using the Western Blot Technique of blood samples from 96 end-stage renal disease patients who were undergoing hemodialysis, and from 100 healthy individuals.

Results. Frequency distribution of the basic apo(a) isoforms calculated by means of the $\chi^{2}$-test has shown that there was no significant statistical difference in distribution among patients on hemodialysis, and healthy carriers $\left(\chi^{2}-0.36, \mathrm{p}<0.548\right.$-for carriers of single apo(a) isoforms, $\chi^{2}-0.10, \mathrm{p}<0.7545$-for carriers of double apo(a) isoforms). The calculated relative risks have demonstrated that apo(a) phenotype was not a risk factor for ASVD in HD patients. Conclusion. Based on the results obtained, it can be concluded that the apo(a) phenotype is not a risk factor for arteriosclerotic vascular disease in patients undergoing hemodialysis.
\end{abstract}

Key words: apo(a) phenotype, ESRD hemodialysis

\section{Introduction}

End-stage renal disease (ESRD) patients undergoing hemodialysis are at an increased risk of arteriosclerotic vascular disease (ASVD). The increased risk is commonly attributed to the traditional risk factors related to ESRD [1]. However, interest for more recent ASVD risk factors, such as the level of lipoprotein(a) and its specific apoprotein(a), has been promoted [2].

Correspondence to: Danica Labudovic, Institute of Medical and Experimental Biochemistry, Faculty of Medicine, Skopje, "50 Divizija" 6, 1000 Skopje, R. Macedonia; Phone: + 38923217 303; Fax: + 3892 3230 431; Mob: + 389702060 70; E-mail: dlabudovic@yahoo.com
Lipoprotein(a) is composed of an LDL particle to which apoprotein(a) has a covalent bonding with S-S bridges. Apoprotein(a) [apo(a)] is a glycoprotein with the so-called Kringle structure (in German kringle means "pretzel"). There is a different number of Kringle IV-type 2 units in various individuals, which are coded by a high polymorph APO(a) gene [3]. The repetition number affects the size of the apo(a) molecule, and the size of this molecule is in an inverse correlation with the $\mathrm{Lp}(\mathrm{a})$ concentration in the people's blood [4].

The apo(a) polymorphism molecule size is detected by using SDS-PAGE and a follow-up of the Western Blot Technique. On a polyacrilamide gel, based on the mobility in relation to Apo B100, 6 apo(a) isomorphs have been separated in humans [5].

The aim of this paper is to determine whether apo(a) phenotype is a risk factor for arteriosclerotic vascular disease in ESRD patients who undergo hemodialysis.

\section{Material and methods}

Examinees. A total of 96 end-stage renal disease (ESRD) patients undergoing dialysis at the Dialysis Ward of the University Clinic of Nephrology in Skopje were included and compared to the control group of 100 healthy individuals. The sole inclusion element for the study was the dialysis treatment, regardless of possible previous kidney disorders which had led to the patients'ending up with hemodialysis.

The two groups, patients and healthy individuals, were first asked to sign an Inform Consent Document for participating in the study.

$\mathrm{K}_{3}$ EDTA plasma blood samples and healthy individuals' serum were taken after a 12 hour (overnight) deprivation of food. The blood samples of the patients were taken from the arteriovenous fistula before the dialysis treatment. The patients were undergoing a regular dialysis regime (3-5 hours three times a week).

Following the low velocity blood centrifugation, the plasma samples were divided in small portions of 1.5 $\mathrm{ml}$ plastic caped Ependorphs and frozen at $-80^{\circ} \mathrm{C}$ until the actual analysis. The fresh serum was used to define the lipid status of the examinees.

Phenotype analysis of apoprotein(a) isomorphs was done by means of the Western Blot Test: first the separation with $3-15 \%$ gradient denaturation SDS-PAGE of Mi- 
ni-Protean II Bio-Rad Vertical System (Bio-Rad Laboratories, Hercules, CA, USA) was done and second, the transfer of proteins onto a nitrocellulose (NC) membrane by electro-blotting with Hoefer TE 22 Mini Tank Transfer Unit which was connected to a circulating bath set up at $+10^{\circ} \mathrm{C}$. The plasma samples were treated with SDS, glycerol, bromophenol-blue and $\beta$-mercaptophenol, and then applied in wells of mini 3-15\% gradient gels. Simultaneously, we applied the commercial standard (Immuno-AG, Vienna, Austria) in two wells of one mini gel. The Apo(a) standard contained 6 apo(a) isomorphs: B, F, S1, S3, S4, >S4. Electrophoresis was leaking into a TRIS-glycerin puffer until the blue front left the gel. With the electro-blotting in the TRIS-glycerin-methanol puffer the proteins were transferred to the NC membrane (S\&S NC; BA 83; $0.2 \mathrm{~mm}$ ) with the above mentioned instruments.

On the third day, the apo(a) isomorphs were immune-chemically visualized by means of Lp(a) phenol-typifying. The details of the complete procedure have already been published [6].

Based on their relative electophoretic SDS-PAGE mobility, compared to ApoB100, and by applying the Utermann nomenclature, the apo(a) isomorphs were separated into $\mathrm{F}, \mathrm{B}, \mathrm{S} 1, \mathrm{~S} 3, \mathrm{~S} 4$ and $>\mathrm{S} 4$ single-band isomorphs and appropriate apo(a) double-banded isomorphs. In case a blob was not visualized at the application place, the phonotype was defined as "null" phenotype [7] (Figure 1).

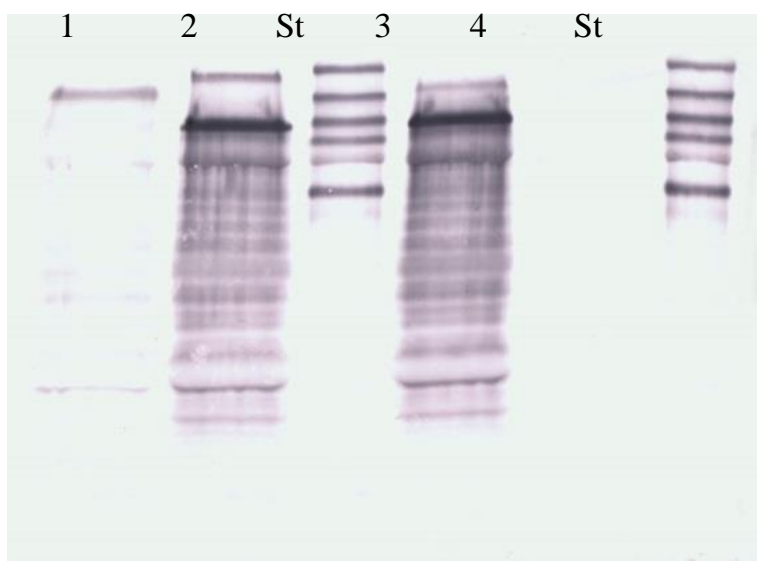

Fig. 1. NC membrane with samples of individuals who carry a single apo(a) isoform (1), double apo(a) isoform (2 and 3), null phenotype (4); St- apo(a) standard (Immuno-Ag)

The molecule mass (MM) of each apo(a) isoform of the samples was calculated by comparing it to apo(a) isoform $\mathrm{MM}$ present in the standard by means of a laser scanner and Image Master Software (Pharmacia Biotechnology, Uppsala Sweden).

According to Utermann et al. $>\mathrm{S} 4, \mathrm{~S} 4$ and $\mathrm{S} 3$ are high molecular apo(a) isoforms which contribute to the low $\mathrm{Lp}$ (a) concentration in the blood, whereas F, B, S1 are low molecular apo(a) isoforms which are responsible for the high $L p(a)$ values in the carriers' blood [6].

\section{Determination of the concentration of lipoprotein(a), apolipoproteins and lipid parameters}

The Lp(a), ApoA-1 and ApoB concentration was determined in a plasma sample with immunonephelometric kit (DADEBehring, Germany of Behring Nephelometer Analyzer). Triacylglycerols and the total cholesterol were determined in a fresh serum with enzymatic CHOD-PAP test kits (Randox Laboratories-UK). HDL-cholesterol was determined by means of an enzymatic test kit in the supernatant that was received after depositing the other lipoproteins with a mixture of phosphowolframat and $\mathrm{MgCl}_{2}$ (Randox Laboratories-UK). LDL-cholesterol was mathematically calculated by means of the Friedewald formula. Glucose was determined by means of the Beckman Glucose Analyzer.

\section{Statistical analysis}

The results obtained are presented as a medium arithmetical value \pm standard deviation (SD); the lipoprotein (a) $[\mathrm{Lp}(\mathrm{a})]$ concentration is also presented as a median. The Student's t-test was used for comparing the age, the concentration of $\operatorname{Lp}(\mathrm{a})$, apoproteins and the parameters of the lipid status between the ESRD patients undergoing hemodialysis and the healthy individuals (control group). $\chi^{2}$-test was used for comparison between the single apo(a) isoforms of the ESRD patients undergoing hemodialysis and the healthy individuals. The statistical analysis was conducted with STATWIN statistical package (Statsoft Inc, Tulsa Oklahoma USA). The $\mathrm{p}<0.05$ value was considered to be statistically significant.

\section{Results}

The clinical characteristics and the data acquired with the laboratory research for HD patients and the control group are presented in Table 1. Increased blood pressure was measured in $27.08 \%$ of the patients, but in none of the control examinees. The positive family anamnesis of kidney diseases was found in $20.83 \%$ of the patients. The duration of the hemodialysis was from 1-36 years ( $9.33 \pm 7.32$ years). Due to the fact that no significant statistical difference in the $\mathrm{Lp}(\mathrm{a})$ concentration was determined between the two genders and the two groups of examinees ( $p<0.12$-patients; $p<0.16$-control group), the results are given as a sum for both genders and for the groups of patients and the controls.

The mean value of the $\mathrm{Lp}$ (a) concentration was moderately increased compared to the control group, but it was statistically insignificant. Table 1 illustrates an increased level of triacylglycerols and hypocholesterolemia in the ESRD patients compared to the control group [7].

The sum distribution of the basic apo(a) isoforms carriers in the patients and the control group, separated by $3-15 \%$ gradient SDS-PAGE isoforms, expressed in percent, is presented in Figure 2. 
Table 1. Clinical characteristics and concentration of lipid status parameters in ESRD HD patients and the control group (healthy individuals)

\begin{tabular}{|c|c|c|c|}
\hline Variables & $\begin{array}{c}\text { ESRD patients } \\
\text { undergoing } \mathrm{HD} \\
n=96\end{array}$ & $\begin{array}{l}\text { Control group } \\
n=100\end{array}$ & $\begin{array}{c}\text { p-value } \\
\text { ESRD pat./ } \\
\text { control group }\end{array}$ \\
\hline Age (years) & $51.59 \pm 13.26$ & $50.69 \pm 12.89$ & n.s. \\
\hline Smokers $(\%)$ & 14.00 & 16.00 & n.s. \\
\hline $\operatorname{NTA}(\%)$ & 27.08 & - & - \\
\hline $\begin{array}{l}\text { Duration of hemodialysis } \\
\text { (years) }\end{array}$ & $9.33 \pm 7.32$ & - & - \\
\hline $\begin{array}{l}\text { Positive family history of } \\
\text { kidney disease }(\%)\end{array}$ & 20.83 & - & - \\
\hline $\begin{array}{l}\mathrm{Lp}(\mathrm{a}) \mathrm{mg} / \mathrm{dl} \\
(\text { median })\end{array}$ & $\begin{array}{l}17.33 \pm 17.44 \\
\quad(9.62)\end{array}$ & $\begin{array}{l}14.14 \pm 9.58 \\
(9.62)\end{array}$ & n.s. \\
\hline $\mathrm{Lp}(\mathrm{a}) \geq 30 \mathrm{mg} / \mathrm{dl}(\%)$ & 12.63 & 7.69 & - \\
\hline $\begin{array}{l}\mathrm{Lp}(\mathrm{a}) \geq 30 \mathrm{mg} / \mathrm{dl} \\
\text { (median) }\end{array}$ & $\begin{array}{l}57.79 \pm 21.28 \\
\quad(53.75)\end{array}$ & $\begin{array}{l}55.49 \pm 18.35 \\
\quad(51.6)\end{array}$ & n.s. \\
\hline ApoA1 (g/L) & $0.98 \pm 0.20$ & $1.19 \pm 0.19$ & 0.001 \\
\hline ApoB (g/L) & $0.871 \pm 0.29$ & $0.81 \pm 0.25$ & n.s. \\
\hline Triacilglycerols (mmo/L) & $1.93 \pm 0.95$ & $1.32 \pm 0.71$ & 0.01 \\
\hline Total cholesterol (mmol/L) & $3.91 \pm 1.04$ & $4.45 \pm 0.89$ & 0.001 \\
\hline HDL- chol. (mmol/L) & $0.822 \pm 0.29$ & $1.22 \pm 0.30$ & 0.001 \\
\hline LDL - chol. (mmol/L) & $2.22 \pm 0.94$ & $2.88 \pm 0.99$ & 0.001 \\
\hline Glycise $(\mathrm{mmol} / \mathrm{L})$ & $5.60 \pm 2.37$ & $5.32 \pm 0.77$ & n.s. \\
\hline
\end{tabular}

The analysis shows that the distribution of the basic apo(a) isoforms carriers between the HD patients and the control group is rather similar. Most frequent are carriers of single apo(a) isoforms $(60 \%)$, and then come the carriers of the double apo(a) isoforms (35\%).
The percent of the "null" phenotype carriers in ESRD patients undergoing hemodialysis $(3.13 \%)$ is lower compared to the control group $(4.95 \%)$, but the difference is statistically insignificant. $\chi^{2}$-test demonstrated that there is no significant statistical difference in the basic

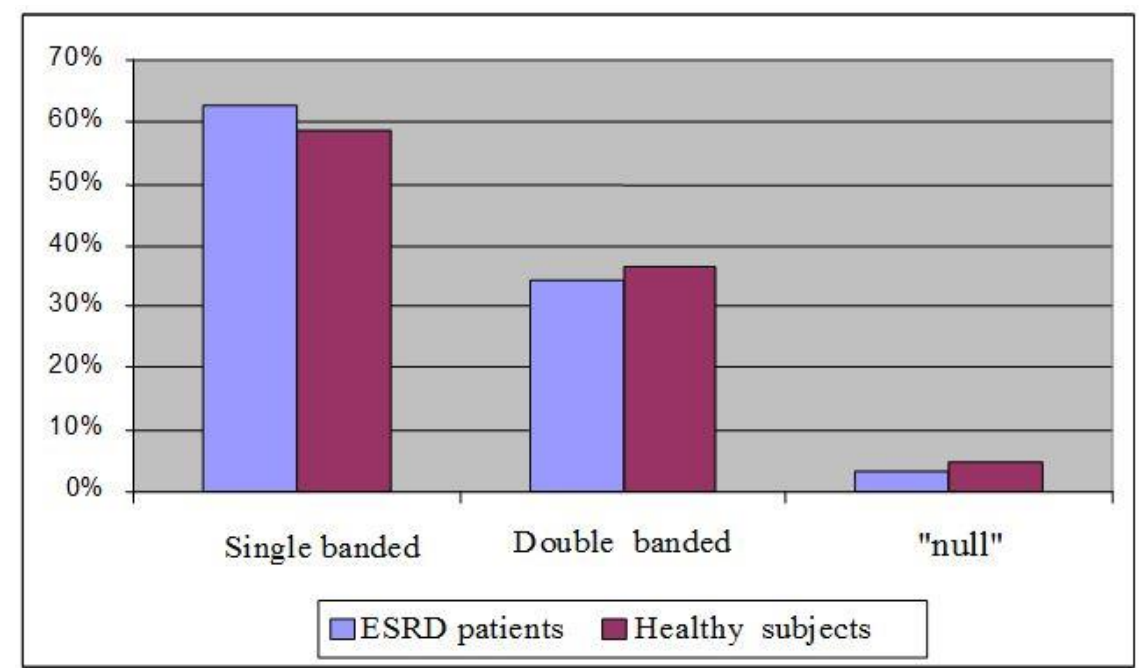

Fig. 2. Frequency distribution of basic apo(a) isoforms (single and double and null phenotypes) in HD patients and control group

apo(a) isoform distribution frequency between patients on hemodialysis and the control group $\left(\chi^{2}-0.36, \mathrm{p}<0.548\right.$ -for the single apo(a) isoform carriers, $\chi^{2}-0.10$, $\mathrm{p}<0.7545$ for the double apo(a) isoform carriers).

Figure 3 (A and B) shows the separated single (A) and double apo(a) isoforms and the carriers of the "null" phenotype for the patients undergoing hemodialysis and the control group.

The Figure shows that high molecular single >S4, S4, apo(a) isoforms were more present in patients undergoing hemodialysis compared to the control group, whereas S3 apo(a) isoforms were less present compared to the control 


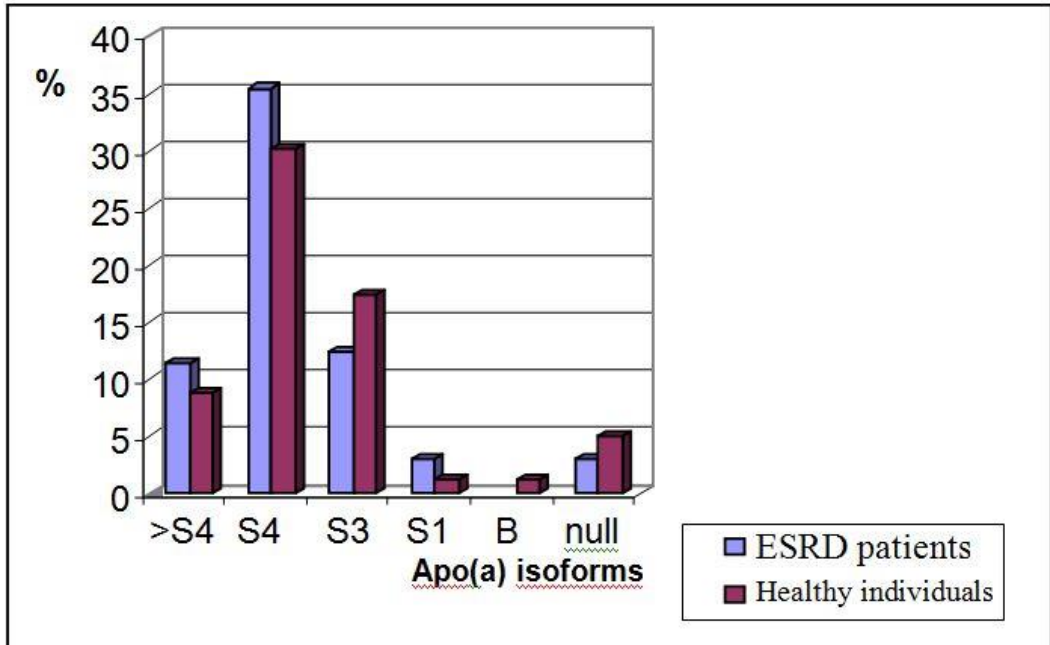

Fig. 3a.

$\%$

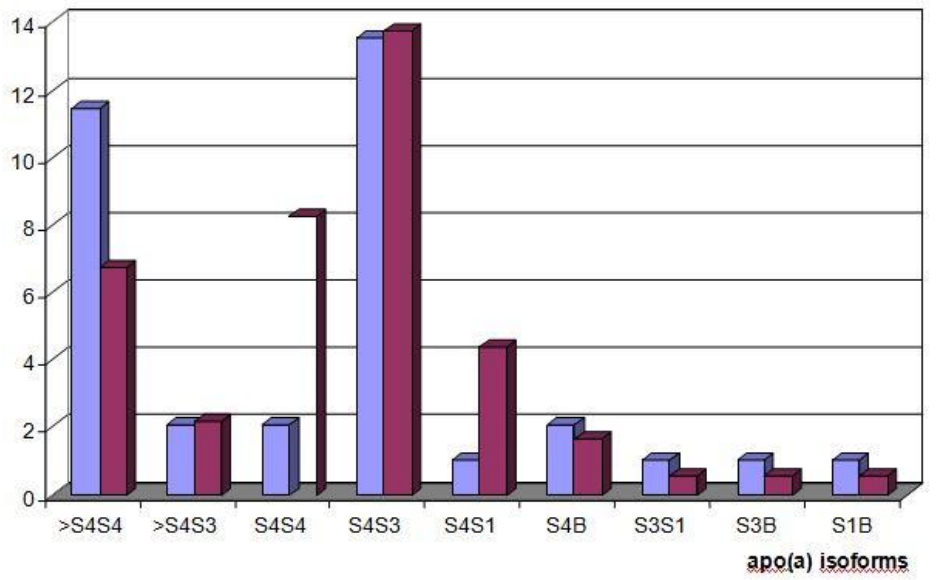

$\square$ ESRD patients $\square$ Healthy individuals

Fig. 3b.

Fig. 3a and 3b. Separated single (A) and double (B) apo(a) isoforms and carriers of null phenotype in HD patients and the control group (in \%)

Table 2. Relative risks of individual apo(a) isoforms in the atherogenesis of HD patients compared to the control group

\begin{tabular}{lccccc}
\hline $\begin{array}{c}\text { Apo(a) } \\
\text { isoforms }\end{array}$ & $>\mathbf{S 4}$ & $\mathbf{S 4}$ & $\mathbf{S 3}$ & $\mathbf{S 1}$ & $\mathbf{B}$ \\
\hline HD(n) & 20 & 47 & 19 & 4 & 3 \\
Control (n) & 23 & 48 & 23 & 3 & 3 \\
OR & 1.82 & 0.69 & 0.94 & 2.59 & 1.92 \\
$95 \%$ CI & $0.90-3.69$ & $0.41-1.16$ & $0.48-1.80$ & $0.48-14.95$ & $0.30-12.21$ \\
RR & 1.65 & 0.84 & 0.95 & 2.53 & 1.90 \\
$95 \%$ CI & $0.96-2.85$ & $0.66-1.71$ & $0.58-1.55$ & $0.58-11.06$ & $0.39-9.21$ \\
p & 0.072 & 0.139 & 0.831 & 0.200 & 0.342 \\
\hline OR-odds ratio; RR-relative risk; 95\% CI-confidence interval; p-statistical significance for \\
the assessed apo(a) relative risks between patients and control group
\end{tabular}

group. However, while scanning the $\mathrm{NC}$ membrane on the LKB Image Scanner, which was used to measure the apo(a) molecular isoform mass, we found that the mean arithmetic value of the $\mathrm{S} 3$ apo(a) isoform molecular mass (611.24 \pm 46.29$)$ in HD patients was statistically signifycantly higher compared to the healthy carriers of the same S3 apo(a) isoform (586.18 \pm 20.85$)$ ( $p>0.0065)$. In carriers of the double high molecular $>\mathrm{S} 4 \mathrm{~S} 4$ apo(a) isoform was found in higher percent $(11.46 \%)$ in patients undergoing hemodialysis compared to the carriers of the same phenotype in the control group (6.72\%). We also found low molecular S1 phenotype carriers both among the patients and among the healthy examinees, whereas low molecular B phenotype was found only in 
the healthy individuals. The $\chi^{2}$-test analysis showed no statistically significant difference in the apo(a) isoform frequency between the two examined groups.

The role of the apoprotein (a) isoforms in the development of atherogenesis in patients undergoing hemodialysis is presented in Table 2 by estimating the relative risks. The values of certain apo(a) isoforms are a result of the sum of single apo(a) isoforms and the predominance of the double apo(a) isoforms.

From the assessed relative risks it can be concluded that none of the apo(a) isoforms is a risk factor in the development and progress of atherogenesis in patients undergoing hemodialysis compared to the relevant control examinees.

\section{Discussion}

The kidneys play an active role in the Lp(a) catabolism. This is why it is considered that the increased Lp(a) concentration in patients undergoing hemodialysis is primarily a result of the decreased clearance $\operatorname{Lp}(\mathrm{a})$, rather than the increased $\mathrm{Lp}(\mathrm{a})$ synthesis, regardless of the apo(a) phenotype [8]. The HD patients have an increased risk for atherosclerotic disease [1,9].

The frequency distribution of apo(a) isoforms for ESRD patients undergoing hemodialysis was very similar to the distribution in the healthy group which was confirmed with the $\chi^{2}$-test and which is in line with the finding of Parsons et al. [10].

In our study we have not found that apo(a) isoforms are a risk factor for atherosclerotic vascular diseases, and in particular, we have not found a relation between the low molecular apo(a) isoforms and CAD, which may be due to the small number of patients on hemodialysis, and low molecular apo(a) isoform carriers. The reference data are contradictory. According to the Choice Study conducted on 871 examinees on HD, there was no connection between apo(a) isoforms and CAD, particularly between the small apo(a) isoforms and atherosclerotic changes in these patients. However, in the same study, by applying the Cox Hazard Population Analysis it was found that patients of both races (white and black race representatives were included), the carriers of the low molecular apo(a) isoforms had a significantly shorter life [2,11]. Kronenberg et al. in a study including 404 randomly selected HD patients found that CAD was associated with low molecular apo(a) phenotype (RR-2.3, $\mathrm{p}=0.0008)$, but not with the high $\operatorname{Lp}(\mathrm{a})$ values in the patient's blood. According to them, the size of the apo(a) molecule and $\mathrm{Lp}$ (a) concentration have a synergetic role in the progress of atherosclerosis in its more advanced stage. In addition, they believe that apo(a) phenotype, which precisely reflects the $\mathrm{Lp}$ (a) level, may be considered to be a marker for those patients who have a more damaged vascular endothelium before the beginning of ESRD; these patients may develop a "galloping" atherosclerosis to follow the beginning of ESRD, thus they need to be more aggressively treated with statins and other medicine which can decrease the $\mathrm{Lp}$ (a) level for at least a little in order to prevent undesired vascular changes [12].

\section{Conclusions}

Based on the results obtained we can say that apo(a) phenotype is not a risk factor for atherosclerotic vascular diseases in patients undergoing hemodialysis. It is most likely that the conventional risk factors (lipid parameters, increased blood pressure, smoking) are still the most responsible factors for ASVD in HD patients.

Conflict of interest statement. None declared.

\section{References}

1. Sarnak MJ, Levey AS, Schoolwerh AC, et al. Kidney disease as a risk factor for development of cardiovascular disease. A statement from the American Heart Association council on kidney in cardiovascular disease, high blood pressure research, clinical cardiology and epidemiology and prevention. Hypertension 2003; 42: 1050-1065.

2. Longenecker JC, Klag MJ, Marcovina SM, et al. High lipoprotein(a) levels and small apoprotein(a) size prospectively predict cardiovascular events in dialysis patients. J Am Soc Nephrol 2005; 16: 1794-1802.

3. Chretien JP, Coresh J, Berthier-Shaad Y, et al. Three singlenucleotide polymorphism in LPA account for most of the increase in lipoprotein(a) level elevation in African Americans compare to European Americans. J Med Genet 2006; 43: 917-923.

4. Kostner KM, \& Kostner GM. Lipoprotein(a): still an enigma? Curr Opinion Lipidol 2002; 13: 391-396.

5. Utermann G. Genetic architecture and evolution of the lipoprotein(a) trait. Curr Opin Lipid 1999; 10: 133-141.

6. Tosheska K, Labudovic D, Alabakovska S, et al. Lipoprotein(a) and apolipoprotein(a) phenotypes in healthy Macedonian children. Bratisl Lek Listy 2007; 108(4-5): 179-183.

7. Labudovic D, Toseska Trajkovska K, Alabakovska S, et al. Lipoprotein(a) and lipid status in ESRD patients on hemodialysis. MMP 2007; (1-3): 16-20.

8. Albers JJ, Koschinski ML, Marcovina SM. Evidence mounts for a role of the kidney in lipoprotein(a) catabolism. Kidney Int 2007; 71(10): 961-962.

9. Shojaei MH, Djelali M, Siassi F, et al. Serum levels of lipoprotein(a) and homocysteine in patients on hemodialysis who take hydroxymethylglutaril-CoA reductase inhibitors, vitamin B6 and folic acid. IJKD 2009; 3: 141-144.

10. Parsons DS, Reaveley DA, Pavitt DV, et al. Lipoprotein(a) levels in those with high molecular weight ano(a) isoforms may remain low in significant proportion of patients with end-stage renal disease. Nephrol Dial Transplant 2003; 18: 1848-1853.

11. Longenecker JC, Klag MJ, Marcovina SM, et al. Small apolipoprotein(a) size predicts mortality in end-stage renal disease. The CHOICE Study. Circulation 2002; 106: 2812-2818.

12. Kronenberg F, Neyer U, Lhotta K, et al. The low molecular weight apo(a) phenotype is an independent predictor for coronary artery disease in hemodialysis patients: A prospective follow up. J Am Soc Nephrol 1999; 10: 1027-1036. 